SMAD, Rev. Eletrônica Saúde Mental Álcool Drog.

2019 abr.-jun.;15(2):12-19

DOI: 10.11606/issn. 1806-6976.smad.2019.151541

www.revistas.usp.br/smad/

Artigo Original

\title{
Estratégias de enfrentamento do craving em dependentes de crack em tratamento em Comunidades Terapêuticas*
}

Paulo Renato Vitória Calheiros ${ }^{1}$

Paulo Rogério Morais ${ }^{1}$

Itamar José Félix-Junior ${ }^{2}$

Leila Gracieli da Silva ${ }^{3}$

Marta de Almeida ${ }^{1}$

\footnotetext{
* Apoio financeiro do Conselho Nacional de Desenvolvimento Científico e Tecnológico (CNPq), Brasil, processo no 402696/2010.

${ }^{1}$ Universidade Federal de Rondônia, Porto Velho, RO, Brasil. ${ }^{2}$ Prefeitura Municipal de Porto Velho, Porto Velho, RO, Brasil.

${ }^{3}$ Faculdade de Ciências Biomédicas de Cacoal, Cacoal, RO, Brasil.
}

\begin{abstract}
Objetivos: identificar e descrever as estratégias de enfrentamento utilizadas perante o craving por usuários de crack que estavam em tratamento internados em Comunidades Terapêuticas. Método: a amostra foi composta por 133 homens. Os instrumentos utilizados foram: Questionário sociodemográfico e rastreio do uso de drogas, Mini-Exame do Estado Mental e uma Escala de Perguntas sobre craving. A análise utilizada foi a estatística descritiva e de frequências para análise exploratória com nível de significância de 5\%. Resultados: os participantes relataram um predomínio da categoria Força de Vontade e de comportamento de Esquiva em resposta ao craving. Conclusão: esses resultados são importantes contribuições na compreensão do enfrentamento do craving, sinalizando a importância do trabalho da prevenção de recaídas, da família e tratamentos eficazes.

Descritores: Estratégias de Enfrentamento; Craving; Tratamento; Comunidades Terapêuticas.
\end{abstract}

\section{Como citar este artigo}

Calheiros PRV, Morais PR, Félix-Junior IJ, Silva LG, Almeida M. Coping strategies of craving in crack-cocaine dependents in treatment in Therapeutic Communities. SMAD, Rev Eletrônica Saúde Mental Álcool Drog. 2019;15(2):12-19. doi: https://dx.doi.org/10.11606/issn.1806-6976.smad.2019.151541 


\title{
Coping strategies of craving in crack-cocaine dependents in treatment in Therapeutic Communities
}

\begin{abstract}
Objectives: to identify and describe the coping strategies used in the face of craving by crackcocaine users who were under treatment in the Therapeutic Communities. Method: the sample consisted of 133 men. The instruments were a socio-demographic questionnaire and on drug use screening, the Mini-Mental State Examination and the scale of questions on craving. The analysis used was descriptive and frequency statistics for exploratory analysis with significance level of $5 \%$. Results: the results demonstrate a predominance of the Willpower category and Dodge in response to craving. Conclusion: these results are important contributions in understanding the coping of craving, signaling the importance of relapse prevention work, of the family and of effective treatments.
\end{abstract}

Descriptors: Coping Strategies; Craving; Treatment; Therapeutic Communities.

\section{Estrategias de afrontamiento en craving de dependiente de crack em tratamiento en Comunidades Terapéuticas}

\begin{abstract}
Objetivos: el objetivo del estudio fue identificar y describir las estratégias de afrontamiento utilizadas frente al craving por consumidores de crack que estaban em tratamiento internados en Comunidades Terapéuticas. Método: la muestra consistió en 133 hombres. Los instrumentos fueron: cuestionario sociodemográfico y la detección del consumo de drogas; Mini Examen del Estado Mental y Craving Cuestionario. El análisis utilizada fue descriptiva y las estadísticas de frecuencia para el análisis exploratorio con un nivel de significación del 5\%. Resultados: los resultados muestran el predominio de la categoría de fuerza de voluntad y el comportamiento de Esquivar en respuesta a las ansias. Conclusión: estos resultados son importantes contribuciones en la comprensión de la cofia antojo, lo que indica la importancia de la labor de prevención de recaídas, la familia y los tratamientos eficaces.
\end{abstract}

Descriptores: Estrategias de Afrontamiento; Craving; Tratamiento; Comunidades Terapéuticas. 


\section{Introdução}

O uso do crack, que é a pasta base de cocaína com bicarbonato de sódio, por via pulmonar (crack), tornou-se um problema de saúde pública no Brasil, dado os efeitos nocivos da substância no organismo e os custos com tratamento(1). O padrão de consumo abusivo, contínuo e repetitivo de crack é provocado pelo craving(2).

A definição de craving ou fissura, como é popularmente conhecido no Brasil, ainda é discutível(3). Para o comitê de especialistas em dependência química da Organização Mundial de Saúde (OMS), o craving pode ser definido como um desejo de repetir a experiência dos efeitos de uma dada substância - formado por componentes cognitivos, afetivos, comportamentais e fisiológicos ${ }^{(4)}$. Outros pesquisadores descrevem o tema como desejo de experimentar os efeitos da droga; forte impulso para utilizar a substância; expectativa de resultados positivos; processo de avaliação cognitiva e processo cognitivo não automático(2-3).

Embora o II Levantamento nacional de álcool e drogas aponte que a proporção de pessoas que experimentaram crack alguma vez na vida seja de $0,1 \%$ entre adolescentes e de 1,3\% nos adultos e o crack não seja a substância ilícita mais consumida no país(5), os efeitos e a intensidade do craving aumentam os problemas relacionados ao uso da droga(2), isso porque a cocaína presente no crack é um potente estimulante do sistema nervoso central, com alto potencial de dependência, haja vista que a quase imediata euforia desencadeada pelo uso reforça e motiva novos episódios de consumo(6). Na dependência de crack, o craving habitualmente é finalizado quando o usuário chega a um esgotamento físico, psíquico e/ou financeiro. 0 seu enfrentamento ganha relevância no tratamento de dependentes de $\operatorname{crack}^{(7)}$.

Em um estudo foram investigadas as estratégias de enfrentamento utilizadas para administrar situações de craving em 35 sujeitos, dependentes de crack segundo os critérios da CID-10(6). Os resultados apontaram que algumas estratégias de enfrentamento estão correlacionadas à motivação para parar de usar crack e também com o tempo de tratamento (internação) e abstinência.

Em um estudo que consistia na predição do craving e o consequente uso ou não da substância em 205 usuários de cocaína inalada e crack em um ambulatório de tratamento, os resultados demonstraram que usuários de cocaína fumada tinham mais episódios de craving do que os usuários de cocaína inalada( ${ }^{(8)}$. Também houve associação significativa entre dependência, em que a frequência de uso predizia mais situações de craving, assim como o intervalo de abstinência predizia menos episódios. Outro ponto importante é que a falta de estratégias eficazes no enfrentamento do craving aumenta significativamente as chances de recaída e de abandono terapêutico.

Em uma pesquisa nacional, os participantes usuários de crack, responderam acerca dos motivos que impulsionaram o início do uso da substância, $58,28 \%$ declararam curiosidade e $29,19 \%$ afirmaram que problemas familiares ou perdas afetivas foram os principais $\operatorname{motivos}^{(9)}$. Esses dados se correlacionam positivamente com as estratégias empregadas por usuários de crack em recuperação para administração do craving.

Acerca das estratégias de enfrentamento, é importante ressaltar que elas se caracterizam como uma resposta ao estresse (comportamental ou cognitiva) com a finalidade de reduzir as suas qualidades aversivas, no caso o uso de crack. A pessoa então pode escolher estratégias de enfrentamento de acordo com seu repertório individual (muitas estratégias podem ser úteis para uma mesma situação) e do reforço que as experiências já trouxeram anteriormente( ${ }^{(6)}$.

Uma grande parcela dos usuários de crack e também de álcool e outras drogas é recebida pelas Comunidades Terapêuticas. As Comunidades Terapêuticas são locais que ofertam acolhimento a usuários de todos os tipos de drogas, geralmente em locais afastados e com caráter residencial transitório, nas quais o tempo de internação varia entre seis e nove meses para adultos. O que habitualmente ocorre nessas instituições é o emprego do modelo religioso-espiritual para todas as substâncias ${ }^{(10)}$ e a resistência ao uso de fármacos para o craving ${ }^{(11)}$.

Destarte, tendo em vista a dificuldade de manejar situações de craving do crack, somada ao elevado número de usuários e à limitação dos tratamentos ofertados, faz-se pertinente pesquisar as estratégias que têm sido empregadas pelos usuários no enfrentamento dessas situações. O objetivo deste estudo é descrever as estratégias utilizadas por dependentes de crack em tratamento em Comunidades Terapêuticas nas principais cidades do estado de Rondônia para administração do craving.

\section{Métodos}

Trata-se de um estudo de caráter descritivoexploratório e análise quantitativa, com recorte transversal e procedimento de campo.

A amostra foi composta por 133 homens que preenchiam os critérios para dependência de cocaína/ crack do DSM-IV-TR ${ }^{(12)}$. Todos estavam internados em comunidades terapêuticas e relataram que o último uso de crack havia sido realizado há mais de seis meses. Os participantes deste estudo embora pudessem se enquadrar nos critérios para dependência de outras 
substâncias psicoativas, o uso de crack foi referido como o principal motivador para a busca de tratamento. Foram excluídos os participantes com escore inferior a 25 pontos no Mini-Exame do Estado Mental e com transtornos ou sintomas psicóticos.

Os dados foram coletados em Comunidades Terapêuticas, previamente mapeadas pela Superintendência Estadual de Políticas sobre Drogas (SEPOAD) de Rondônia, nas principais cidades do estado, a saber: Porto Velho, Cacoal, Ji-Paraná, Rolim de Moura, Vilhena e Ariquemes.

Para a coleta de dados, utilizaram-se os seguintes instrumentos: 1)Termo de Consentimento Livre e Esclarecido; 2)Questionário de identificação elaborado pelos próprios pesquisadores com base em instrumentos de rastreio do uso de drogas, composto por itens abertos e fechados que abordam características sociodemográficas e socioeconômicas; 3)Mini-Exame do Estado Mental (MEEM) ${ }^{(13)}$ para avaliar quadros de deficit cognitivos, que podem comprometer a coleta de dados e ser afetados pelo uso pesado de crack; e 4)aEscala de perguntas sobre Craving, adaptada com base no "Questionário de crenças sobre o uso de drogas"(14). O questionário é composto por uma parte quantitativa e uma qualitativa. A primeira parte compreende 50 questões, nas quais são exemplificadas situações e o sujeito é convidado a imaginar vivenciando-as e, em seguida, ele deve responder qual a força de vontade de utilizar a droga e qual a chance de ter uma recaída. A parte qualitativa foram questões referentes à recusa de utilizar a substância e quais as estratégias utilizadas.

A coleta de dados se deu após o mapeamento das Comunidades Terapêuticas destinadas ao atendimento de dependentes químicos no estado de Rondônia e contato prévio com os responsáveis. A partir da autorização dos responsáveis, a escolha dos participantes se deu aleatoriamente pelos números pares, a partir do número de pessoas em tratamento no local. As entrevistas foram individuais, em um espaço físico adequado, indicado pela própria instituição. As sessões duraram em média 30 minutos e a coleta ocorreu durante o período de julho de 2012 e abril de 2013.

As informações coletadas foram organizadas em uma planilha eletrônica no programa Statistical Package for the Social Sciences (SPSS), versão 20.0. A análise dos dados constou de testes estatísticos descritivos e de frequências para análise exploratória dos dados. O nível de significância foi de $5 \%$.

O projeto foi aprovado pelo Comitê de Ética em Pesquisa da Universidade Federal de Rondônia (Parecer No: 367.336; CAAE 03725212.8.0000.5300), e todos os participantes receberam esclarecimentos e assinaram o TCLE antes do preenchimento dos instrumentos.

\section{Resultados}

A média de idade da amostra foi de 31 anos $(D P=8,76)$. Outros dados sóciodemográficos se encontram na da Tabela 1.

Tabela 1 - Composição da amostra e perfil social

\begin{tabular}{lc}
\hline Descrição & $\mathbf{n} \%$ \\
\hline Estado civil & \\
Solteiro (a) & 9874 \\
Casado (a)/mora com parceira (o) & 1813,5 \\
Separado (a)/divorciado (a) & 1712,8 \\
Possui Filhos & \\
Sim & 7556,8 \\
Não & 5743,2 \\
Grau de escolaridade & \\
Não sabe ler ou escrever & 21,5 \\
Ensino fundamental incompleto & 6050,4 \\
Ensino médio completo & 1410,5 \\
Ensino médio incompleto & 2115,8 \\
Ensino fundamental completo & 2015 \\
Superior incompleto & 64,5 \\
Superior completo & 32,3 \\
Situação de emprego atual & \\
Desempregado & 6750,4 \\
Turno integral (35h ou mais de trabalho por semana) & 3627,1 \\
Trabalho informal/autônomo & 2115,8 \\
Meio turno (<35h por trabalho) & 96,8 \\
Passou noite na rua nos últimos seis meses & \\
Sim & 2720,3 \\
Não & 10679,7 \\
\hline &
\end{tabular}

Em relação à avaliação da escala craving, utilizou-se o teste qui-quadrado para avaliar se existiam diferenças estatisticamente significativas entre as categorias. Foi detectada diferença estatisticamente significativa entre as respostas $\left(x^{2}=176,3, d f=8, p<0,01\right)$.

A Tabela 2 traz os dados acerca da pergunta sobre o que fez no momento do craving. Em resposta, a categoria força de vontade apresentou frequência maior do que as demais categorias, seguida pelas respostas referentes a pensamentos relacionados à família, sendo estatisticamente igual à categoria pensamentos negativos em relação à droga, mas diferente das demais categorias de respostas. A frequência de respostas para as categorias pensamentos religiosos, fez uso de álcool ou outras substâncias, sem dinheiro para comprar a droga, atividades sociais não diferiu entre elas.

Tabela 2 - Estratégias utilizadas para enfrentar o desejo

\begin{tabular}{lcc}
\hline Categorias das respostas & N & $\%$ \\
\hline Força de vontade & 58 & 43,6 \\
Pensamentos relacionados à família & 15 & 11,3 \\
Pensamentos negativos acerca da droga & 12 & 9,0 \\
Pensamentos religiosos & 7 & 5,3 \\
Sem dinheiro para comprar a droga & 4 & 3,0 \\
Atividades sociais & 2 & 1,5 \\
Fez uso de álcool ou outras substâncias & 2 & 1,5 \\
Outros & 6 & 4,5 \\
Total & 133 & 100 \\
\hline
\end{tabular}

A Tabela 3 corresponde ao ato comportamental relacionado ao momento de recusa. A análise constatou 
diferença estatisticamente significativa entre as respostas $\left(x^{2}=43,3, d f=5, p<0,01\right)$. A categoria que difere das demais respostas foi busca de auxilio profissional, e demais categorias foram estatisticamente iguais.

Tabela 3 - Estratégias utilizadas para evitar o uso da substância

\begin{tabular}{lcc}
\hline Categorias das respostas & $\mathbf{n}$ & $\%$ \\
\hline Atividades cotidianas & 20 & 15,0 \\
Atividades religiosas & 16 & 12,0 \\
Atividades laborais /sociais & 16 & 12,0 \\
Dormir & 15 & 11,3 \\
Uso de outras drogas & 12 & 9,0 \\
Busca de auxílio profissional & 4 & 3,0 \\
Outros & 20 & 15,0 \\
Total & 133 & 100 \\
\hline
\end{tabular}

Já na Tabela 4, verificou-se diferença estatisticamente significativa entre as respostas $\left(x^{2}=41,6, d f=6, p<0,01\right)$. A categoria decisão para mudar o comportamento é estatisticamente maior do que procurar desempenhar outras atividades, medo e preferir outra substância. Decisão de mudar o comportamento e sem desejo no momento são estatisticamente iguais. A categoria desempenhar outras atividades é estatisticamente maior à preferência por outra substância

Tabela 4 - Motivos que o fizeram recusar o uso da substância

\begin{tabular}{lcc}
\hline Categorias das respostas & N & \% \\
\hline Decisão de mudar o comportamento de uso & 27 & 20,3 \\
Sem desejo no momento & 17 & 12,8 \\
Restrição ambiental ou social & 17 & 12,8 \\
Procurou desempenhar outras atividades & 12 & 9,0 \\
Medo & 7 & 5,3 \\
Preferia outra substância & 4 & 3,0 \\
Outros & 21 & 15,8 \\
Não responderam & 28 & 21,1 \\
Total & 133 & 100 \\
\hline
\end{tabular}

$\mathrm{Na}$ Tabela 5, houve diferença estatisticamente significativa entre as respostas $\left(x^{2}=67,5 ; d f=8\right.$; $\mathrm{p}<0,01)$. As categorias pensamentos afetivos e uso de outras substâncias não diferem entre si, mas são estatisticamente superiores às demais categorias.

Tabela 5 - Estratégias que o ajudaram a recusar o uso da substância

\begin{tabular}{lcc}
\hline Categorias das respostas & N & $\%$ \\
\hline Pensamentos afetivos & 28 & 21,1 \\
Pensamentos religiosos & 17 & 12,8 \\
Pensamentos negativos em relação à droga & 16 & 12 \\
Atividades para distração & 10 & 7,5 \\
Sem desejo no momento & 4 & 3 \\
Uso de outras substâncias & 3 & 2,3 \\
Busca por auxílio de terceiros & 1 & 0,8 \\
Outros & 22 & 16,5 \\
Não responderam & 32 & 24,1 \\
Total & 133 & 100 \\
\hline
\end{tabular}

\section{Discussão}

Em relação às características sociodemográficas, a amostra possui características semelhantes ao que é referido em outros estudos. De modo geral, temos que os usuários de crack são, em sua maioria, homens, solteiros, pouco escolarizados, com idade entre 18 e 35 anos e que não têm emprego/renda fixa(6,15-16). Esses dados corroboram com o perfil descrito na Pesquisa Nacional Sobre o Uso de $\operatorname{Crack}^{(10)}$, que sinalizou a população usuária de crack como significativamente vulnerável socialmente.

A amostra foi particularmente constituída por homens, uma vez que houve dificuldades em encontrar locais de tratamentos para mulheres no estado. Havia apenas três instituições que atendiam mulheres e todas elas com um número maior de pessoas em tratamento. Além de apontar a carência de locais para o tratamento da dependência química na população feminina do estado de Rondônia, o baixo número de instituições que atendem mulheres sugere que as especificidades nos cuidados que devem ser oferecidos no tratamento de mulheres com quadros de dependência química pode ser um fator que desmotiva dirigentes de comunidades terapêuticas a oferecer tratamento para essa parcela das pessoas com problemas relacionados ao uso de substâncias. Por conta desse fator, não foi possível fazer uma análise comparativa entre homens e mulheres acerca dos objetivos do estudo. Destaca-se o fato do aumento do uso dessa substância por mulheres e que elas procuram pelo tratamento mais cedo, muitas por conta das complicações ou quando engravidam(17).

Quanto às estratégias que os participantes relataram usar para enfrentamento do craving, foram apresentadas as categorias de respostas para pergunta O que você disse para si mesmo para vencer o desejo? (Tabela2), a resposta predominante foi a força de vontade com 43,6\% $(n=58)$. Esse dado demonstra que as estratégias usadas para controle do craving são muito particulares a cada usuário e ao contexto, de modo que a mesma estratégia pode funcionar com um e fracassar com outro(2).

O conceito de autoeficácia refere-se às percepções que os indivíduos elaboram sobre si mesmos ${ }^{(18)}$. A elaboração dessas percepções pode ser utilizada como dispositivo para alcançar objetivos, obtendo controle sobre o próprio ambiente, compondo os mecanismos psicológicos da motivação. Nesse sentido, a cognição desempenha função na capacidade das pessoas de construir a realidade e autorregularem-se ${ }^{(19)}$. A autorregulação, nesse caso, está relacionada com a força de vontade para mudar o comportamento de uso e consequentemente uso de drogas, e não como recurso principal para lidar com o craving, como relatado pelos participantes aqui investigados. 
Ao verificar o que fizeram em vez de utilizar o crack (Tabela3), pôde-se constatar que os indivíduos se engajaram em atividades religiosas, como ir para a igreja, cantar, ler a Bíblia, e atividades cotidianas, como interagir com a família, praticar esportes, assistir a filmes. Assim, na amostra estudada, observou-se como um adjuvante na recusa ou superação do craving o comportamento religioso e o papel da família. Destaca-se o fato de os dados terem sido coletados com pessoas que estavam em tratamento em CTs de cunho religioso, o que tem sido ponto de discussão sobre a eficácia desse modelo de tratamento, que trabalha com diferentes perspectivas, tais como os 12 passos, em que estratégias de enfrentamento são ditadas pelo trabalho religioso/espiritual(20). É importante que o tratamento integre modelos de prevenção e orientação ao restante da família, como o Programa de Fortalecimento de Famílias (Strengthening Families Program) ${ }^{(21)}$.

Discute-se acerca da eficácia da modalidade de tratamento/intervenção e do quanto esse modelo pode interferir nas estratégias utilizadas pelos indivíduos para manejar situações de craving, conforme sinalizado na Tabela 3. Problematiza-se acerca das intervenções empregadas por Comunidades Terapêuticas no estado de Rondônia, uma vez que tais instituições são majoritariamente de cunho religioso, sendo que houve um aumento acentuado no número dessas instituições, não sendo acompanhado pela contratação de especialistas para lidar e manejar com o craving sentido pelos pacientes.

Quanto às estratégias que contribuíram para recusar a substância (Tabela5), 23,1\% dos entrevistados recorreram a pensamentos relacionados à família, como o medo de deixá-los desamparados e o medo de desapontar os pais ou cônjuge. Essa categoria foi estatisticamente superior à categoria dos pensamentos religiosos. Esse dado pode indicar que os pensamentos relacionados à família atuam como um fator protetivo para evitar o uso da substância, entretanto é importante avaliar se a família oferece o suporte necessário e se não é um ambiente de risco.

Os resultados descritos na Tabela 5 indicam que as estratégias de enfrentamento dos entrevistados foram diversas, destacando-se o fato de muitos participantes terem como recurso estratégico seus próprios pensamentos. Os pensamentos foram subdivididos em afetivos e religiosos, e os negativos em relação às drogas, e eles assumem uma característica comum a muitos usuários que tentam parar ou controlar o uso levando em conta a família e a religião. Isso pode se dar, pois antes de entrar em tratamento a pessoa que é altamente religiosa ou espiritual pode ter a capacidade de visualizar eventos de vida e fatores de estresse através de um esquema de controle pela religião/espiritualidade, assim é possível que através da religião, da condenação do uso, a pessoa reconstrua seu mundo(22).

Em um estudo, usuários de crack se tornaram visivelmente motivados a aderirem ao tratamento quando percebem que poderão ter seus laços familiares reconquistados(23). Outro estudo(24) corrobora o argumento de que o suporte social está associado às necessidades subjetivas do indivíduo e, além disso, está relacionado com a capacidade de suporte emocional da família e amigos na redução do estresse e na resolução de conflitos interpessoais. Acerca disso, independente do modelo de tratamento estudado ser o de internação, fica nítido que quanto mais tempo de isolamento e de dias de abstinência, menores serão os eventos de craving(25), por conta disso o suporte social deve ser integrado e estimulado no tratamento. Essa interação entre o usuário e a sociedade, que o espera fora do tratamento, pode contribuir para menos recaídas, contribuindo para a manutenção da abstinência desejada. O suporte social ajuda na interação entre o usuário e a sociedade, podendo essa influência mútua ajudar no seu tratamento, contribuindo para a manutenção da abstinência desejada. O ideal é que a família adote uma postura colaborativa para contribuir na recuperação do dependente de drogas. Desse modo, o craving, que pode atrapalhar o tratamento, pode ser superado mais facilmente e rapidamente com o auxílio da família.

\section{Conclusões}

O presente estudo elencou as principais estratégias de enfrentamento do craving empregadas por dependentes em tratamento em Comunidades Terapêuticas localizadas no estado de Rondônia. Os dados obtidos neste trabalho evidenciam a necessidade de se trabalhar estratégias de enfrentamento no decorrer do tratamento, uma vez que o papel da família foi muito lembrado pelos participantes. Trabalhar mais os aspectos familiares pode significar maior poder para enfrentar a dura rotina de se manter abstinente, o que é cobrado por muitos que esperam do usuário uma mudança perante o uso do crack. Além do mais, esses achados poderão ser utilizados no delineamento de cursos de capacitação e aprimoramento às instituições, como as Comunidades Terapêuticas de cunho religioso, que ofertam serviços de atenção a pessoas com transtornos por uso de substâncias, no planejamento de tratamentos contextualizados e eficazes no enfrentamento da dependência de crack, beneficiando, dessa forma, o indivíduo em recuperação e toda a rede assistencial e comunitária envolta.

O estudo contém algumas limitações, como o fato de não abranger dados com mulheres e o grande número de pessoas que não souberam responder. Esses problemas devem ser sanados em estudos posteriores, 
que demonstrem como se dá o processo de tratamento dentro de comunidades terapêuticas e sua relação com os eventos de craving.

Os resultados de estudos com o objetivo de conhecer as estratégias de enfrentamento ao craving antes do tratamento podem contribuir para o desenvolvimento de protocolos de tratamento e para o melhor planejamento de atividades terapêuticas em toda a rede de atenção ao usuário de drogas, principalmente no modelo de tratamento de internação em Comunidades Terapêuticas de cunho religioso, que sofrem críticas justamente por não possuírem muitos profissionais especializados. Também contribuem para entender o fenômeno do tratamento em regiões vulneráveis ao abuso de crack, como é o caso do estado de Rondônia, localizado próximo aos países produtores e exportadores da coca, Peru e Bolívia principalmente, onde a substância pode chegar mais pura.

Importante ressaltar ainda que conhecer as estratégias de manejo e enfretamento do craving é essencial para que a pessoa em recuperação possa se apropriar daquelas que Ihe forem pertinentes, assim como é importante reconhecer as situações de risco para que se consiga evitá-las ou enfrentá-las. Tanto o trabalho da Prevenção de Recaídas quanto o Treino de Habilidades Sociais são potencializados quando o paciente traz suas experiências exitosas anteriores em ter superado o craving e a oferta de uso. Todas essas práticas em Comunidades Terapêuticas devem ser repensadas e requerem o auxílio de profissionais capacitados e especializados em dependência química, que devem ser somados ao suporte familiar - fator que ainda é pouco trabalhado de forma profissional e que ainda destoa da realidade vigente nos tratamentos ofertados, não apenas no estado aqui investigado, mas na maioria do país.

\section{Referências}

1. Oliveira LGD, Nappo SA. Caracterização da cultura de crack na cidade de São Paulo: padrão de uso controlado. Rev Saúde Pública. 2008;42(4):664-71.

2. Chaves TV, Sanchez ZM, Ribeiro LA, Nappo SA. Fissura por crack: comportamentos e estratégias de controle de usuários e ex-usuários. Rev Saúde Pública. 2011;45(6):1168-75.

3. Araújo RB, Oliveira MDS, Pedroso RS, Miguel AC, Castro MDGTD. (2008). Craving e dependência química: conceito, avaliação e tratamento. J Bras Psiquiatr. 2008;57(1):57-63.

4. United Nations International Drug Control Programme (UNDCP), World Health Organization (WHO). Informal Expert Committee on the Drug-Craving Mechanism. United Nations International Drug Control Programme and World Health Organization. Technical report series 5: 92-54439 T 1992.

5. Laranjeira R, Madruga CS, Pinsky I, Caetano R, Mitsuhiro SS, Castello G. II Levantamento Nacional de Álcool e Drogas (LENAD) - 2012 [Internet] São Paulo: INPAD/UNESP; 2013 [Acesso 7 ago. 2016]. Disponível em: http://inpad.org.br/wp-content/uploads/2014/03/ Lenad-II-Relatório.pdf

6. Araujo RB, Pansard M, Boeira BU, Rocha NS. As estratégias de coping para o manejo da fissura de dependentes de crack. Clin Biomed Res. 2010;30(1) 36-43.

7. Marques AC, Seibel SD. O craving. In: Seibel SD, Toscano JR, organizadores. Dependência de drogas. São Paulo: Atheneu; 2001. p. 239-48.

8. Da Silveira DX, Doering-Silveira E, Niel M. Jorge MR. Predicting craving among cocaine users. Addictive Behav. 2006;31(12):2292-7.

9. Bastos FIPM, Bertoni N. Pesquisa Nacional sobre o uso de crack: quem são os usuários de crack e/ou similares do Brasil? Quantos são nas capitais brasileiras? [Internet] Rio de Janeiro (RJ): ICICT/FIOCRUZ; 2014 [Acesso 8 ago 2016] Disponível em: http://www.arca. fiocruz.br/handle/icict/10019

10. Damas FB. Comunidades Terapêuticas no Brasil: expansão, institucionalização e relevância social. Rev Saúde Pública Santa Catarina. 2013;6(1):50-65

11. Fracasso L. Características da comunidade terapêutica. In: Guerra A, et al. (Org.). Curso nacional de aprendizado à distância: aspectos básicos no tratamento da dependência química. Brasília: SENAD. 2002. p. 88-99.

12. American Psychiatric Associaion APA (2002). Manual diagnóstico e estatístico de transtornos mentais: texto revisado. DSM-IV-TR. Porto Alegre (RS): Artmed; 2002.

13. Bertolucci PH., Brucki S, Campacci SR, Juliano, Y. O mini-exame do estado mental em uma populaçäo geral: impacto da escolaridade. Arq. Neuropsiquiatr. 1994; 52(1):1-7

14. Wright FD. Craving Belief Questionnaire and Relapse Prediction Scale. In: Beck AT, Wright FD, Newman CF, Liese BS. Cognitive Therapy of Substance Abuse. New York (NY): Guilford Press; 1993.

15. Oliveira LGD, Nappo SA. Caracterização da cultura de crack na cidade de São Paulo: padrão de uso controlado. Rev Saúde Pública. 2008;42(4):664-71.

16. Oliveira GP. (2011). Crack e Recaída: os principais motivos que levam os usuários de crack a recaírem após tratamento para dependência química [Internet]. Porto Alegre (RS): Universidade Federal do Rio Grande do Sul; 2011 [Acesso 13 ago 2016]. 
Disponível em: http://www.lume.ufrgs.br/bitstream/ handle/10183/34045/000789984.pdf?sequence $=1$

17. Santos AMCC. Articular saúde mental e relações de gênero: dar voz aos sujeitos silenciados. Ciência Saúde Coletiva. 2009;14(4):1177-82

18. Bzuneck JA. As crenças de autoeficácia e o seu papel na motivação do aluno. In: E. Boruchovith $E$, Bzuneck JÁ, (Orgs.). A Motivação do Aluno: Contribuições da Psicologia Contemporânea. Petrópolis (RJ): Editora Vozes; 2001. p. 116-33.

19. Freire SD, Oliveira MDS. Auto-eficácia para Abstinência e Tentação para Uso de Drogas Ilícitas: Uma Revisão Sistemática revisão sistemática. Psicologia: Teoria e Pesquisa. 2011; 27(4):527-36.

20. Longshore D, Anglin MD, Conner BT. Are religiosity and spirituality useful constructs in drug treatment research?. J Behav Health Serv Res. 2009;36(2):177-88. 21. Kumpfer $\mathrm{KL}$, Alvarado R, Whiteside HO. Family-based interventions for substance use and misuse prevention. Substance Use Misuse. 2003;38(11-13):1759-87.

22. Larson DB, Swyers JP, McCullough ME. (Eds.). Scientific research on spirituality and health: $A$ consensus report. Rockville (MD): National Institute for Healthcare Research; 1998

23. Longshore D, Anglin MD, Conner BT. Are religiosity and spirituality useful constructs in drug treatment research?. J Behav Health Serv Res. 2009;36(2):177-188.

24. Almeida RB, Caldas MT. As dificuldades e Sucessos no tratamento do Usuário de crack. Neurobiologia. 2011;74(2): 99-112.

25. Pinho PH, Oliveira, MAD, Almeida MMD. A reabilitação psicossocial na atenção aos transtornos associados ao consumo de álcool e outras drogas: uma estratégia possível?. Rev Psiquiatr Clín. 2008;35(suppl 1):82-8.

26. Lappann NC, Machado JSDA, Tameirão FV, Benjamim MLN. Craving pelo crack nos usuários em tratamento no centro de atenção psicossocial. SMAD. Rev Eletrônica Saúde Mental Álcool e Drogas. 2015;11(1):19-24. 